\title{
Défis et complications liés aux lentilles sclérales - Reconnaissance, étiologie et gestion
}

\author{
L'auteur est Daddi Fadel DOptom, FSLS, FBCLA \\ Publié en 2020 par Dougmar Publishing Group Inc., Dundas (Ontario); 386 pages.
}

William Ngo, DO, Ph. D., FAAO

Centre de formation et de recherche oculaires, École d'optométrie et de sciences de la vision,

Université de Waterloo

\section{I}

es lentilles sclérales sont généralement utilisées pour traiter les problèmes cornéens complexes et les maladies de la surface oculaire. Jusqu'à la dernière décennie, la pose et la prescription des lentilles sclérales appartenaient à des groupes très spécialisés en optométrie. Cependant, au fil du temps, les progrès réalisés dans la fabrication des matériaux perméables au gaz et les techniques de pose ont entrainé une forte augmentation de la popularité des lentilles sclérales. Cette amélioration de l'accessibilité a permis à un plus grand nombre de cliniciens de prendre en charge et de gérer des maladies plus complexes de la surface cornéenne et oculaire, ce qui se traduit directement par des avantages et des soins accrus pour les patients. Aujourd'hui, l'art et la science de la pose des lentilles sclérales évoluent plus rapidement que jamais.

Malgré la popularité des lentilles sclérales, l'exercice de pose de ce type de lentille reste néanmoins intimidant. Le temps qu'il pourrait être nécessaire de consacrer à la résolution de problèmes de pose et de gestion des complications peut s'avérer frustrant et coûteux pour le praticien et le patient. Ce livre, Scleral Lens Issues and Complications : Their Recognition, Etiology, and Management (Défis et complications liés aux lentilles sclérales - Reconnaissance, étiologie et gestion), est une publication opportune qui allie des preuves scientifiques à des perles cliniques, et aborde toute une gamme de sujets liés aux complications des lentilles sclérales.

Les deux premiers chapitres de l'ouvrage expliquent comment les colorants vitaux (fluorescéine et vert Lissamine) sont utilisés pour évaluer et diagnostiquer la qualité de la pose des lentilles sclérales et le lien qui existe avec le profil scléral. Le texte est accompagné d’illustrations de haute qualité pour mettre en évidence les concepts décrits.

Les deux chapitres suivants, qui constituent l'essentiel du livre, traitent des problèmes et des complications liés à la pose et à l'usure des lentilles sclérales. Cette section est présentée de façon systématique pour chaque affection, en commençant par la technique d'examen (p. ex., niveau d'éclairage de la lampe à fente, technique d'éclairage, grossissement), suivie des symptômes et des signes d'affections, de leur étiologie et de leur gestion. La rédaction de cette section est très abrégée, mais efficace, ce qui en fait une source de consultation rapide. Ces problèmes et complications, y compris ceux liés à une mauvaise pose des lentilles, à leur manipulation, à leur entretien et à leur conformité, sont résumés dans le chapitre suivant.

La section qui suit traite des facteurs qui influent sur la vision et le confort. Ce chapitre est utile pour la résolution de problèmes, car il englobe près de 30 situations différentes où le confort et/ou la vision sont altérés par le port de lentilles sclérales (p. ex., vision embrouillée en milieu de journée, mouillabilité médiocre, astigmatisme résiduel). Ces conditions sont également résumées dans des tableaux pour faciliter la consultation. En ce qui concerne l'optique, le chapitre qui suit traite de diverses optiques de surface disponibles pour les lentilles sclérales, comme 
la correction torique de l'astigmatisme, les lentilles à foyers multiples pour la presbytie, les conceptions guidées par front d'ondes pour gérer les aberrations et le prisme pour la correction de la vision binoculaire. Enfin, le livre se termine par une section sur le nettoyage des lentilles sclérales, les processus de désinfection, de manipulation et d'entreposage des divers types de lentilles. Ce chapitre est utile pour mettre en évidence les points importants qui doivent être communiqués au patient.

Dans l'ensemble, ce livre résume presque tous les problèmes et les complications qu'on rencontre lorsqu'on porte des lentilles sclérales. Il servira de ressource utile pour un large éventail de praticiens, qu'il s'agisse de ceux qui commencent à poser des lentilles sclérales, ou de ceux qui ont plus d'expérience avec ce type de lentilles et qui veulent simplement un livre de référence rapide. 